\title{
Effects of large scale bridging in load controlled fatigue delamination of
}

\section{unidirectional carbon-epoxy specimens}

E. Farmand-Ashtiani, J. Cugnoni, J. Botsis ${ }^{1}$

École Polytechnique Fédéral de Lausanne (EPFL), LMAF-STI, Lausanne CH-1015, Switzerland

Abstract: Fatigue delamination growth in composites is accompanied by large scale bridging (LSB) that yields important toughening effects. However, the extent of this mechanism depends on the laminate geometry rendering its modeling a challenging task. This work presents a combined experimental/numerical study on characterization of specimen thickness dependence of LSB in fatigue delamination. Double cantilever beam specimens of different thicknesses ( $h=2,4$ and $8 \mathrm{~mm}$ ), equipped with arrays of multiplexed fiber Bragg grating sensors, are subjected to mode I fatigue loads.

Measured strain data with the sensors are employed to identify the bridging tractions and subsequently compute the energy release rate (ERR) due to the bridging as well as the ERR at the crack tip. The obtained results confirm that fatigue delamination growth strongly depends on the specimen geometry when LSB prevails. It is shown that both the extent of bridging and critical ERR at failure increase by increasing the specimen thickness while the maximum bridging traction at the crack tip is found independent of the specimen geometry. The identified traction-separation relations serve to establish a power correlation, between the crack growth rate and ERR at the crack tip which is independent of the specimen thickness.

Keywords: A. Polymer-matrix composites B. Delamination, B. Fatigue, C. Fiber bridging, Scale effects 


\section{Introduction}

Delamination under fatigue loads is widely recognized as a critical failure mode of fiber-reinforced laminates. Significant progress has been achieved on this matter over the past few decades as recently reviewed in [1]. In particular, a wealth of literature exists on modeling and experimentation of fatigue delamination based on the fracture mechanics concepts [1-6]. Yet, a thorough characterization of governing mechanisms on delamination growth under cyclic loading is still lacking. Moreover, the standardized experimental methods for characterization of fatigue delamination, such as ASTM D6115, are limited only to the onset of delamination. These limitations are primarily due to the complexity of damage events that take place in the wake of the crack during delamination growth. An important damage mechanism that can accompany fatigue delamination propagation in fibrous laminates is crack bridging by intact fibers. Bridging fibers effectively reduce the stress level at the vicinity of the crack tip and consequently contribute to crack growth resistance: under fatigue loads, development of the bridging zone affects the rate of crack growth and can lead to crack deceleration and even crack arrest. Hence, assessment of bridging effects is of particular importance in the characterization of fatigue delamination in composite laminates. In the literature, several semi-empirical relationships between the cyclic loading parameters and delamination growth rate are proposed. These models often employ a Paris-Erdogan relation that relates the rate of crack growth to the applied cyclic energy release rate (ERR) or stress intensity factor through a power law relation [1]. However, the applicability of such relations is often limited to the experimental conditions in which they are established. Moreover, such an approach cannot predict crack growth deceleration while the total applied ERR increases. 
In contemporary approaches, bridging effects are described by a distribution of tractions over the crack faces. The relationship between the bridging tractions and corresponding crack opening displacements (COD), called traction separation relation, serve in computational methods to model delamination [4, 7-9]. A direct experimental assessment of crack-bridging tractions in fatigue delamination is, however, a challenging task, as it requires precise local measurements of strains and displacements along the bridging zone. As a consequence, the modeling efforts accounting for bridging do not always rely on such local measurements and for convenience bridging tractions are often estimated from the global response of the specimen [10-12]. Yet, distribution and intensity of bridging in fatigue delamination may differ from bridging in monotonic delamination fracture [13-15]. Moreover, when large scale bridging (LSB) prevails, the extent of bridging can be influenced by the laminate geometry.

In previous works [16-18], specimen thickness dependence of LSB in delamination and fracture of unidirectional laminates under monotonic loads is reported. Here, an iterative method [13] is adopted to identify the bridging traction contribution in the fracture of fiber-reinforced polymer matrix composites: it is based on distributed strain measurements, with multiplexed fiber Bragg grating (FBG) sensors, along the crack propagation direction and subsequent inverse identification of bridging tractions using parametric finite element (FE) modeling. Employing such an approach in the analysis of monotonic fracture demonstrates that both the bridging zone length and steady state ERR significantly increase by increasing the specimen thickness while the crack initiation ERR, as well as the maximum bridging traction at the crack tip, are independent of the specimen geometry [16-18]. A recent micromechanical analysis of LSB supports the specimen thickness dependence of bridging during fracture [19]. 
Although progress has been reported on understanding bridging phenomena, studies on load-controlled fatigue delamination are very scarce. Thus, in this work, LSB effects and the specimen thickness dependence in fatigue delamination are investigated. Unidirectional carbon epoxy specimens of different thicknesses, equipped with integrated arrays of multiplexed FBGs, are subjected to mode I force-controlled fatigue loads. Measured strain data with FBG sensors are employed to identify the bridging tractions and subsequently quantify the crack tip ERR as well as the ERR due to bridging. The results allow elucidating bridging effects in delamination growth.

\section{Materials and methods}

\subsection{Specimens}

Unidirectional carbon epoxy laminates with thicknesses of $h=2,4$ and $8 \mathrm{~mm}$ are fabricated by stacking prepreg layers of SE 70 from Gurit ST ${ }^{\mathrm{TM}}$. An initial crack starter is introduced at the mid-plane of the laminates by inserting a $60 \mathrm{~mm}$ long and $13 \mu \mathrm{m}$ thick release film from Aerovac ${ }^{\circledR}$. The laminates of different thicknesses are cured using the same standard procedure suggested by the prepreg manufacturer so that the variation of fiber volume fraction between the composite plates is less than $1 \%$ and the maximum variation in thickness for any given specimen does not exceed $0.1 \mathrm{~mm}$. Double cantilever beam (DCB) specimens are prepared by cutting the cured composite plates into $25 \mathrm{~mm}$ wide beams and bonding steel loading blocks (10x25x10 mm) to the beams end. One side of each specimen, normal to the crack plane, is painted white and marked with black thin lines at every millimeter to provide a clear image of the crack tip location during the tests. The specimens with $h=2$ and $4 \mathrm{~mm}$, are equipped with optical fibers (SM28, $125 \mu \mathrm{m}$ in diameter), each containing 10 multiplexed FBG sensors. The sensors are equally spaced at $3 \mathrm{~mm}$ center to center and each one has a gauge length of 
$1 \mathrm{~mm}$. In case the of the $2 \mathrm{~mm}$ thick specimen, the optical fiber (with the coating removed) is embedded four layers away from the crack plane. For the $h=4 \mathrm{~mm}$ specimen, the array of sensors is bonded to the specimen surface using a liquid cyanoacrylate instant adhesive (Loctite ${ }^{\circledR} 401$ ). In both cases, the optical fibers are aligned parallel to the carbon fibers' direction and centered in the width of the specimen. The exact position of FBGs along the longitudinal axis of the beam is measured using the optical low-coherence reflectometry technique with a step length of $25 \mu \mathrm{m}$. The following elastic constants are used for the numerical analysis: longitudinal modulus $E_{z}=120.2 \mathrm{GPa}$, transverse moduli $E_{y}=E_{x}=7.3 \mathrm{GPa}$, shear moduli $G_{z y}=G_{z x}=$ 3.9 GPa and Poisson's ratios $v_{z y}=v_{z x}=0.28, v_{y x}=0.48$ (Fig. 1) [20].

\subsection{Fatigue testing}

Mode I fatigue tests are performed using an Instron ${ }^{\circledR}$ machine equipped with a $500 \mathrm{~N}$ load cell in force control. A schematic of the fatigue testing configuration is shown in Fig. 1a. In total, nine specimens (three specimens per laminate thickness) are tested. Prior to fatigue testing, the specimens are ramp loaded in displacement control (3 $\mathrm{mm} / \mathrm{min}$ ) to initiate a natural crack from the end of the insert film. The load at crack initiation under monotonic loading of each specimen is reduced by $20 \%$ and applied in the subsequent fatigue experiment as the maximum cyclic load. Loading consists of a sinusoidal waveform with a frequency of $f=2 \mathrm{~Hz}$ and a minimum to maximum load ratio of $R=0.5$. The experiments are terminated at total fracture of the specimen which is the result of unstable crack growth at the end of each fatigue test. Crack propagation is followed throughout the tests using a high resolution CCD camera. The rate of crack growth, $\Delta a$ / $\Delta N$, is determined from the crack length data, $a$, at every millimeter and number of elapsed cycles, $N$. It is experimentally observed that the specimens behave 
linearly elastic in loading/unloading cycles with negligible energy dissipation. The peak values of load, $P$, and displacement, $\Delta$, are acquired at each fatigue cycle and subsequently combined with the corresponding crack length data to obtain the maximum cyclic values of the total applied ERR, $G_{t}$, as follows:

$$
G_{t}=G_{I, t i p}+G_{I, b}=\frac{P^{2}}{2 b} \frac{d C}{d a}
$$

Here $b$ is the width and $C=\Delta / P$ is the compliance of the specimen. To obtain smooth data for subsequent differentiation, the measured crack length data and corresponding compliance values are fitted to the following power expression: $C=B a^{n}$ where $B$ and $n$ are the fitting constants. Axial strains along the crack propagation direction are monitored by means of the integrated multiplexed FBG sensors. The initial Bragg wavelengths, $\lambda_{B 0}$, are between 1520 and $1565 \mathrm{~nm}$ (spaced by $5 \mathrm{~nm}$ ) with a bandwidth of $1.5 \mathrm{~nm}$. The Bragg wavelengths emitted during the fatigue loading are detected using the Micron Optics SM130 ${ }^{\circledR}$ interrogator with a frequency of $15.8 \mathrm{~Hz}$. It is experimentally observed that the Bragg peaks only shift in response to fatigue crack growth and do not split up. The latter indicates a uniform strain field on the short gratings used. Hence, considering the axial strain, $\varepsilon_{z}$, as the dominant strain component in the optical fiber, the measured shifts of Bragg wavelength, $\Delta \lambda_{B}$, are converted to axial strains as follows: $\Delta \lambda_{B i} / \lambda_{B 0, i}=\left(1-p_{e}\right) \varepsilon_{z, i}$, where $i=1, \ldots, 10$, indicates an FBG sensor along the optical fiber and $p_{e}$ is the effective photo-elastic constant, equal to 0.2148 for the optical fibers used herein. Subsequently, the measured strains are expressed in the local crack tip coordinate system, as previously described in [13], to obtain a quasi-continuous strain distribution for inverse identification of bridging tractions in fatigue. 


\subsection{Identification of the bridging tractions}

To identify the bridging tractions, FE models representing the DCB specimens of each thickness, at the crack lengths of interest, are built in Abaqus ${ }^{\circledR}$ Standard v 6.12. The maximum cyclic strain values are taken as the objective data for the identification procedure, consequently the boundary conditions representing the maximum cyclic loading are considered in the identification models. Only one arm of the specimen is modeled due to the symmetry of the specimen and loading configuration. Quadratic plane strain elements (Abaqus ${ }^{\circledR}$ CPE8R) are employed to discretize the models. At least 12 elements are used in the thickness of the specimen arms and the mesh is locally refined around the crack tip (with the radial length of elements at the crack tip equal to $12.5 \mu \mathrm{m})$. The crack tip singularity $(1 / \sqrt{r})$ is modeled by collapsing the elements at the tip to triangles and shifting their mid side nodes to $1 / 4$ of the element's edge. In total, 8864 to 45082 elements (from the thinnest to the thickest specimens) are used to discretize the models. Fiber bridging is represented by a parametric surface traction applied to the specimen arms normal to the crack plane [13, 16-18]:

$$
\sigma_{b}(z)=\sigma_{\max } e^{-\gamma z}\left(1-z / z_{\max }\right), \quad 0 \leq z \leq z_{\max }
$$

Here $z$ is the distance from the crack tip; $\sigma_{\max }$ is the maximum bridging traction at the crack tip; $Z_{\text {max }}$ is the length of the bridging zone and $\gamma$ is a parameter introduced to account for the nonlinear rate of traction decay along the bridging zone (Fig. 1b). The strains given by the parametric FE model at the position of the nodes corresponding to the sensors location, $\varepsilon_{F E M}$, and the strain data from FBGs, $\varepsilon_{E X P}$, interpolated linearly at the same locations as the nodes of the FE model, are implemented in an error vector 
defined as: $F\left(\sigma_{\text {max }}, Z_{\text {max }}, \gamma\right)=\left\|\frac{\varepsilon_{F E M}-\varepsilon_{E X P}}{\bar{\varepsilon}_{E X P}}\right\|$, where $\bar{\varepsilon}_{E X P}$ is a reference strain value for normalization that is set equal to the mean value of the measured strains. The error norm $F$ serves as the objective function in a non-linear least-squares optimization scheme using a trust-region reflective Newton optimization algorithm built-in in the commercial software Matlab $^{\circledR}$. To identify the optimal bridging traction parameters $\left(\sigma_{\max }, z_{\max }, \gamma\right)$, the objective function is iteratively minimized with several random initial values chosen in the following ranges: $0 \leq \sigma_{\max } \leq \sigma_{\mathrm{m}}$, where $\sigma_{\mathrm{m}}$ is the strength of the matrix, $0 \leq z_{\max } \leq a-a_{0}$, where $a_{0}$ is the length of the crack starter and $0 \leq \gamma \leq 1$. These multiple minimization processes converge to the same results, independent of the initial choice of parameters. This unique set $\left(\sigma_{\max }, z_{\max }, \gamma\right)$ is considered as the solution of the optimization scheme.

The corresponding traction separation relations for each thickness, $\hat{\sigma}_{b}(\delta)$, are constructed by combining each $\sigma_{b}(z)$ with the corresponding values of COD, $\delta(z)$. The later values can be obtained using the weight function for an orthotropic DCB specimen [21]. However, since numerical integrations are necessary in this case, $\delta(z)$, are readily obtained from the optimized FE simulations for simplicity. Note that simulations with linear and non-linear geometrical effects show a difference of $4 \%$ on the bridging traction calculations.

Relations $\hat{\sigma}_{b}(\delta)$ for each thickness serve to numerically compute the ERR of the bridged cracks along appropriate contours, as follows:

$$
J_{t}=J_{I, t i p}+J_{I, b}=J_{I, t i p}+\int_{0}^{\delta_{\max }} \hat{\sigma}_{b}(\delta) d \delta
$$


Here the total ERR, $J_{t}$, is calculated by considering a far-field contour, $\Gamma_{t}$, encompassing the crack tip and entire bridging zone and $J_{I, t i p}$ represents the crack tip ERR obtained with a contour integral local to the crack tip, $\Gamma_{\text {tip }}$, as illustrated in Fig. $1 c$. Parameter $\delta_{\max }$ denotes the opening displacement at the end of the bridging zone (i.e. $\left.\delta\left(z=Z_{\text {max }}\right)\right)$. Thus, in the analysis of ERR presented herein, the term $G$ refers to the maximum cyclic ERR values experimentally obtained with the compliance calibration method (Eq. 1) and $J$ refers to the numerically computed ERR using the contour $J$ integral and the identified bridging tractions (Eq. 3).

\section{Results and discussion}

Representative experimental data of crack length plotted against number of cycles are shown in Fig. 2. The date in Fig. 2 demonstrate that crack propagation consists of an initially high crack growth rate (phase I) that evolves into a long period of slow crack propagation (phase II) which ends by rapid crack growth (phase III) and unstable fracture.

Based on these experimental observations and physical reasoning, the reported delamination fatigue process is envisioned as follows (Fig. 1a): upon loading, damage develops in the crack tip process zone as well as in the bridging zone behind the crack tip with different growth rates. When damage in the crack tip process zone reaches a critical level, local failure takes place and the crack grows in this zone. In parallel, crack advance generates interface failure and new bridging fibers close to the crack tip as well as failure of bridging fibers in the tail of the bridging zone. The process of damage accumulation in both zones is repeated until specimen failure. 
Representative specimens for each thickness and the evolution of the specimens' compliance versus crack length during the fatigue delamination tests are shown in Fig. 3a and 3b, respectively. The slope of these curves are used to calculate the applied maximum cyclic ERR, $G_{t}$, as described in Section 2.2. The measured strain data with an array of 10 multiplexed FBG sensors during the entire fatigue experiment are demonstrated in Fig. 4a for the specimen with $h=2 \mathrm{~mm}$. Each curve represents the evolution of the strains imposed on an individual sensor by cyclic loading. Note that the sensors are loaded in compression due to their position with respect to the neutral axis of the beam (Section 2.1). For the subsequent analysis of bridging effects on fatigue delamination, the maximum values of strain, $\varepsilon_{z}$, are extracted as shown in Fig. 4b.

Due to the force-controlled loading condition, the applied maximum $G_{t}$ to each specimen monotonically increases due to the increase in crack length throughout the fatigue test. The latter is demonstrated in Fig. 5 together with the corresponding resistance curves measured in monotonic delamination tests [16]. The fatigue ERR curves are initially close but diverge progressively up to the failure of each specimen. For a given specimen thickness, the applied ERR is lower than the corresponding monotonic delamination resistance until a critical value at which the specimen fails. As shown in Fig. 5, such critical values are nearly equal to the plateau level of the R-curves in the corresponding monotonic tests.

Figure 6 shows the rate of fatigue crack growth, $\Delta a / \Delta N$, in representative specimens (data of Fig. 2) of different thicknesses plotted against the applied total maximum ERR, $G_{t}$. The three phases of crack propagation, marked in Fig. 2, can also be distinguished here. Namely, crack deceleration (phase I), constant crack speed (phase II) and crack acceleration (phase III) terminated by specimen fracture. Such crack 
growth behavior, also observed elsewhere [13, 22], is attributed to the development of LSB during fatigue, which reduces the ERR at the crack tip in phases I and II, despite the monotonic increase in the applied global ERR throughout the test. Such behavior implies that the presence of the bridging zone and its evolution produces different levels of shielding on the crack tip in these two phases.

The results presented in Fig. 6 demonstrate significant scaling effects on fatigue delamination in the present composite specimens: for a given value of the applied $G_{t}$, crack growth rates become lower with the increase in specimen thickness. Moreover, a higher ERR, at specimen fracture, is achieved in the thicker samples, thus the constant crack growth rate phase (i.e. phase II) becomes longer by increasing specimen thickness. Given the same damage mechanisms (matrix and/or interface failure, fiber bridging and fiber fracture) in delamination of specimens with different thicknesses, the experimental observations can be explained by assuming a more pronounced bridging effect in fatigue delamination of the thicker specimens. To investigate such a hypothesis, the bridging tractions in representative specimens with different thicknesses are determined using the iterative identification of bridging tractions described in Section 2.3. Such an identification scheme requires the analysis of a completely developed bridging zone in each specimen. Yet, the crack bridging mechanism evolves during the entire fatigue delamination growth.

From the results presented in Figs 2 and 6, the successive phases of bridging zone development in a given specimen can be interpreted as follows:

- At the early part of phase I, the bridging zone is negligible and crack growth occurs relatively fast. Upon continued load, the bridging zone increases in size giving rise to slower crack speed. Hence, during this phase, the rate of increase of bridging 
tractions, $\mathrm{d} G_{I, t i p} / \mathrm{d} a$, is larger than the rate of the applied ERR, $\mathrm{d} G_{\mathrm{t}} / \mathrm{d} a$, and $G_{I, t i p}$ decreases with crack growth.

- In phase II, the crack grows at a constant speed while the ERR continues to increase due to increasing crack length (with load controlled conditions) and bridging zone size. The constant crack speed in phase II suggests that the increase in the applied ERR is equal to the increase in the resistance due to bridging. i.e. $\mathrm{d} G_{t} / \mathrm{d} a \approx \mathrm{dG}_{I, b} / \mathrm{d} a$ and $G_{I, t i p}$ remains constant, i.e. $\mathrm{d} G_{I, t i p} / \mathrm{d} a=0$.

- At the end of phase II, the crack starts to accelerate leading to specimen fracture (phase III). This is due to the diminishing rate of bridging zone growth and the acceleration of damage growth in the process zone, rendering additional toughening due to fiber bridging insignificant and all additional energy is spent on crack tip propagation $\mathrm{d}_{t} / \mathrm{d} a \approx \mathrm{dG}_{I, t i p} / \mathrm{d} a$. Eventually complete failure occurs leading to the specimen fracture. Based on the aforementioned sequence of crack propagation, it is assumed that bridging is fully developed at the end of phase II. Thus, the measured strains at the onset of crack acceleration (i.e. end of phase II), are considered for the identification of bridging tractions.

Following the procedure outlined in Section 2.3, the identified bridging parameters are as follows: $\sigma_{\max }=1.15 \mathrm{MPa}, Z_{\max }=14 \mathrm{~mm}, \gamma=0.058 \mathrm{~mm}^{-1}$ for the specimen with $h$ $=2 \mathrm{~mm}$, and $\sigma_{\max }=1.21 \mathrm{MPa}, \mathrm{Z}_{\max }=25 \mathrm{~mm}$ and $\gamma=0.035 \mathrm{~mm}^{-1}$ for the specimen with $h=4 \mathrm{~mm}$. While the traction at the crack tip, $\sigma_{\max }$, is found to be practically the same for the two specimens of different thicknesses, the bridging zone length, $z_{\max }$, and the nonlinear decay parameter $\gamma$, vary considerably with $h$. These findings suggest that specimen thickness dependence of bridging parameters in load controlled fatigue 
delamination follows the same trend as previously reported in the case of monotonic delamination [16-18], though the identified bridging parameters in fatigue differ from the corresponding values in monotonic delamination [16].

Based on these results, the bridging tractions parameters in the DCB specimen with $h=8 \mathrm{~mm}$ are determined by considering $\sigma_{\max }$ as a constant, independent of $h$.

Parameters $\gamma$ and $Z_{\max }$ are obtained by assuming the same scaling relationships found in monotonic delamination [16-18]. Namely, $z_{\max }=C_{1} h+C_{2}$ (where $C_{1}, C_{2}$ are fitting parameters) and $\gamma h=$ const . The complete set of data are shown in Fig. 7.

As mentioned earlier, during phase III, the growth of the bridging zone is insignificant. Consequently, the form of $\hat{\sigma}_{b}(\delta)$ remains the same and $G_{I, b}$ is considered constant $\mathrm{d} G_{I, b} / \mathrm{d} a \approx 0$ (Eq. 1) with all additional energy spent on crack growth with $\mathrm{d} G_{I, t i p} / \mathrm{d} a>0$. Thus using $\hat{\sigma}_{b}(\delta)$ and the applied maximum force at specimen fracture, $J_{I, t i p}$ and $J_{I, b}$ (Eq. 3) are calculated and the results are compared with the measured $G_{t}$ (Eq. 1) for the specimens with the three thicknesses considered in this study. The data in Fig. 9 demonstrate that the calculated total ERR (Eq. 3) and the measured ERR (Eq. 1) at failure are nearly the same. Thus, the identified traction-separation relations implemented in this study can predict well the ultimate failure of the specimens under fatigue, lending support to the adopted identification approach. Furthermore, specimen fracture takes place when the crack tip ERR reaches the interlaminar crack initiation toughness of the present composite material measured in monotonic delamination $J_{I, t i p} \approx G_{I c} \approx 300 \pm 30 \mathrm{~J} / \mathrm{m}^{2}$ [16] (Fig. 9). 
In addition, using the identified traction, $\hat{\sigma}_{b}(\delta)$, the evolution of $J_{I, t i p}$ in phase III can be calculated. Figure 10a shows experimental crack speed data vs. the $J_{I, t i p}$ values identified at several crack lengths in this phase as well as the values at the onset of fatigue delamination. The results demonstrated in Fig. 10a suggest a power relation independent of the specimen thickness and crack length, i.e., $\Delta a / \Delta N=A\left(J_{I, t \text { tip }}\right)^{n}$ where $\mathrm{A}=9.69 \times 10^{-17} \mathrm{~mm} /\left(\mathrm{J} / \mathrm{m}^{2}\right)^{n}$ and $n=5.63$ are fitting parameters. Note that the values of these parameters are similar to recently reported data regarding fatigue delamination in other composites $[14,23]$. It is stated earlier that in phase II, $J_{I, t i p}$ remains constant. Thus $J_{I, b}$ during this phase can be expressed as, $J_{I, b}=J_{t}-J_{I, t i p}$. Replacing $J_{t}$ with the experimental values of $G_{t}$, and using the calculated values of $J_{I, t i p}, J_{I, b}$ is estimated for several vales of crack advance as shown in Fig. 10b. Also shown in Fig.10b are the values of $J_{I, b}$ in phase III, taken equal to the identified values at the transition between phases II and III. The data in phase I are interpolation values between the zero crack advance and the start of phase II.

\section{Conclusions}

1. Load controlled fatigue delamination is accompanied by LSB and significantly depends on the specimen thickness resulting in crack deceleration and eventual specimen fracture at a critical ERR almost equal to the corresponding steady state ERR under monotonic delamination.

2. The identified bridging tractions suggest that the maximum bridging traction at the crack tip is independent of the three tested specimens with thicknesses of 2, 4 and $8 \mathrm{~mm}$. 
However, fatigue delamination in thicker specimens is accompanied by a longer bridging zone, in which the bridging tractions decay with a lower rate.

3. The scaling relationships between the specimen thickness and the bridging parameters, derived previously in the case of monotonic delamination [16, 17], can be effectively used to predict thickness effects on bridging parameters in fatigue.

4. The identified values of ERR at the crack tip and the corresponding crack growth rate data suggest a power relation independent of the specimen thickness and crack length.

Acknowledgement: The authors acknowledge the financial support from the Swiss National Science Foundation under Grant 200020_149721.

\section{References}

[1] Bak BL, Sarrado C, Turon A, Costa J. Delamination under fatigue loads in composite laminates: a review on the observed phenomenology and computational methods. Appl Mech Rev 2014;66(6):060803.

[2] Hojo M, Matsuda S, Tanaka M, Ochiai S, Murakami A. Mode I delamination fatigue properties of interlayer-toughened CF/epoxy laminates. Compos Sci Technol 2006;66(5):665-675.

[3] Shivakumar K, Chen H, Abali F, Le D, Davis C. A total fatigue life model for mode I delaminated composite laminates. Int J Fatigue 2006;28(1):33-42.

[4] Turon A, Costa J, Camanho P, Dávila C. Simulation of delamination in composites under high-cycle fatigue. Compos Part A- Applied S. 2007;38(11):2270-2282.

[5] Stelzer S, Brunner A, Argüelles A, Murphy N, Pinter G. Mode I delamination fatigue crack growth in unidirectional fiber reinforced composites: Development of a standardized test procedure. Compos Sci Technol 2012;72(10):1102-1107. 
[6] Sans D, Renart J, Costa J, Gascons N, Mayugo J. Assessment of the influence of the crack monitoring method in interlaminar fatigue tests using fiber Bragg grating sensors. Compos Sci Technol 2013; 84:44-50.

[7] Bao G, Suo Z. Remarks on crack-bridging concepts. Appl Mech Rev 1992;45:1-16.

[8] Mirzaei B, Sinha A, Nairn J. Measuring and modeling fiber bridging: Application to wood and wood composites exposed to moisture cycling. Compos Sci Technol 2016;128:65-74.

[9] Nairn JA. Analytical and numerical modeling of R curves for cracks with bridging zones. Int J Fracture 2009;155(2):167-181.

[10] Zhang J, Peng L, Zhao L, Fei B. Fatigue delamination growth rates and thresholds of composite laminates under mixed mode loading. Int J Fatigue 2012;40:7-15.

[11] Gregory JR, Spearing SM. A fiber bridging model for fatigue delamination in composite materials. Acta Mater 2004;52(19):5493-5502.

[12] Gregory JR, Spearing SM. Constituent and composite quasi-static and fatigue fracture experiments. Compos Part A- Applied S 2005;36(5):665-674.

[13] Stutz S, Cugnoni J, Botsis J. Studies of mode I delamination in monotonic and fatigue loading using FBG wavelength multiplexing and numerical analysis. Compos Sci Technol 2011;71(4):443-449.

[14] Donough M, Gunnion A, Orifici A, Wang C. Scaling parameter for fatigue delamination growth in composites under varying load ratios. Compos Sci Technol 2015;120:39-48.

[15] Yao L, Alderliesten R, Zhao M, Benedictus R. Bridging effect on mode I fatigue delamination behavior in composite laminates. Compos Part A- Applied S. 2014;63:103-109. 
[16] Farmand-Ashtiani E, Cugnoni J, Botsis J. Specimen thickness dependence of large scale fiber bridging in mode I interlaminar fracture of carbon epoxy composite. Int J Solids Structures 2015;55:58-65.

[17] Pappas G, Botsis J. Intralaminar fracture of unidirectional carbon/epoxy composite: experimental results and numerical analysis. Int J Solids Structures 2016;85:114-124. [18] Manshadi BD, Vassilopoulos AP, Botsis J. A combined experimental/numerical study of the scaling effects on mode I delamination of GFRP. Compos Sci Technol 2013; 83: 32-39.

[19] Canal LP, Pappas G, Botsis J. Large scale fiber bridging in mode I intralaminar fracture. An embedded cell approach. Compos Sci Technol 2016;126:52-59.

[20] Farmand-Ashtiani E, Alanis D, Cugnoni J, Botsis J. Delamination in cross-ply laminates: Identification of traction-separation relations and cohesive zone modeling. Compos Sci Technol 2015;119:85-92.

[21] Massabò R, Brandinelli L, Cox B. Mode I weight functions for an orthotropic double cantilever beam. Int J Eng Sci. 2003;41(13):1497-1518.

[22] Austin T, Singh M, Gregson P, Powell P. Characterisation of fatigue crack growth and related damage mechanisms in FRP-metal hybrid laminates. Compos Sci Technol. 2008;68(6):1399-1412.

[23] Ishbir C, Banks-Sills L, Fourman V, Eliasi R. Delamination propagation in a multidirectional woven composite DCB specimen subjected to fatigue loading. Compos Part B-Eng 2014;66:180-189. 


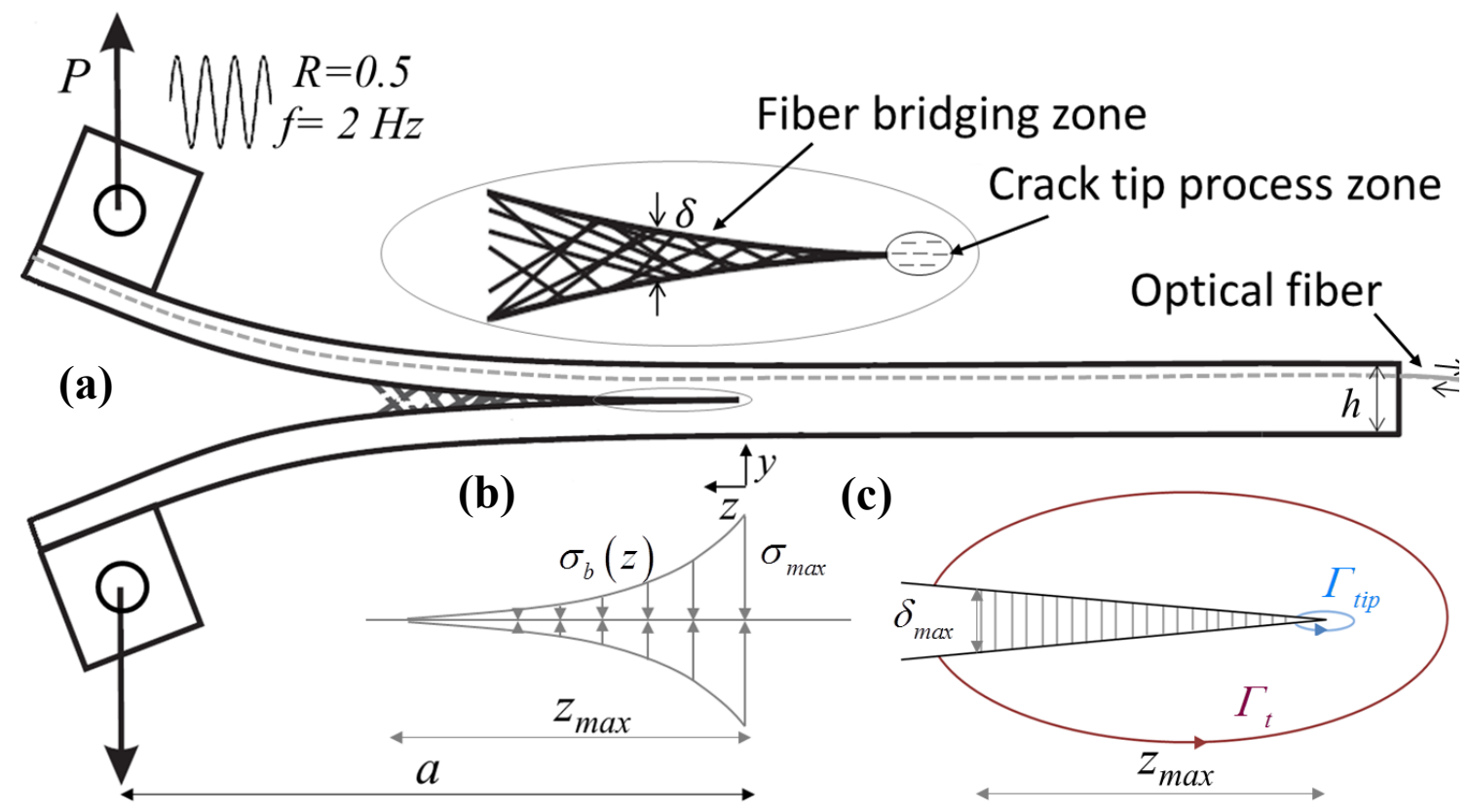

Fig. 1. Schematics representations of: (a) the experimental setup and integrated optical sensors, (b) distributed bridging tractions along the crack, (c) $J$-integral contours associate with the rack tip $\Gamma_{\text {tip }}$ and the entire process zone $\Gamma_{t}$.
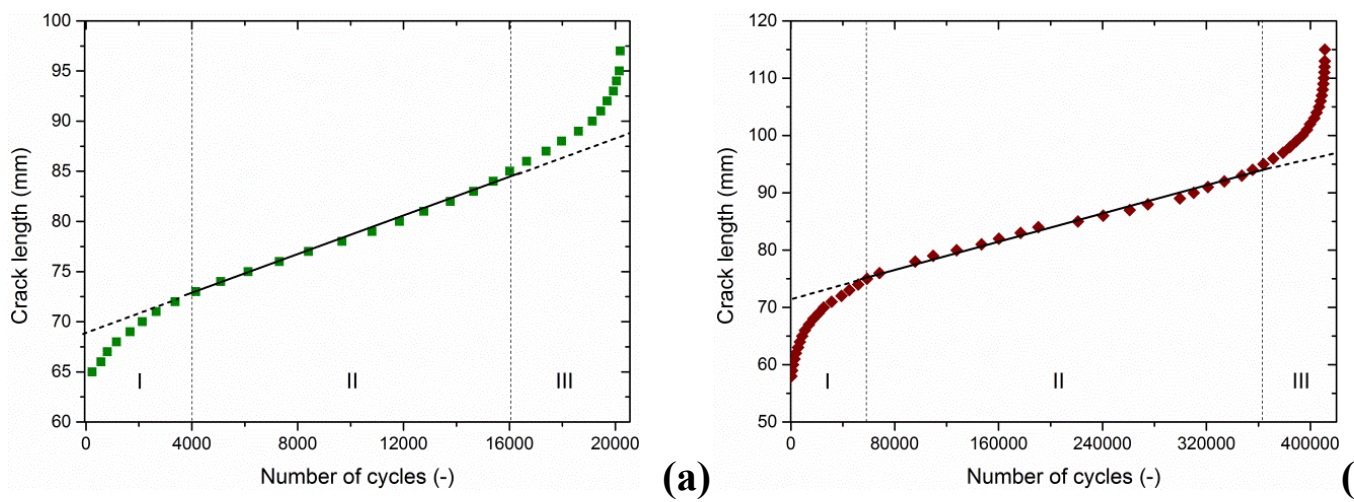

(b)

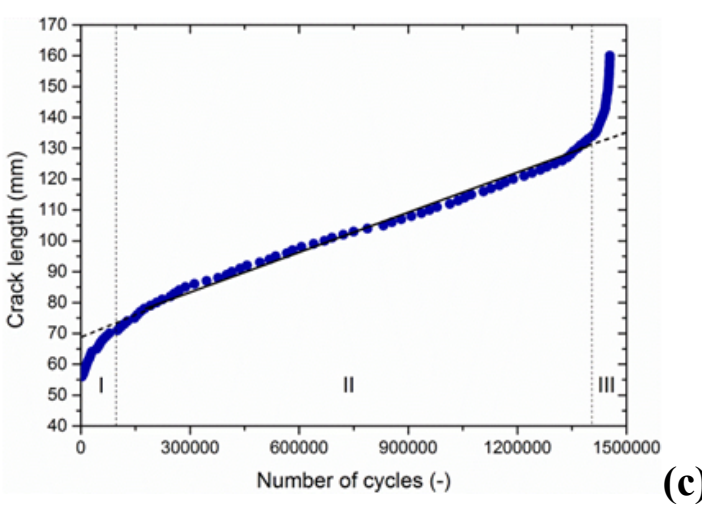

Fig. 2. Representative fatigue crack growth curves indicating three distinct stages of fatigue delamination, (I) crack growth deceleration, (II) constant growth rate (III) crack growth acceleration for: (a) $h=2 \mathrm{~mm}$, (b) $h=4 \mathrm{~mm}$, (c) $h=8 \mathrm{~mm}$. See text for details. 


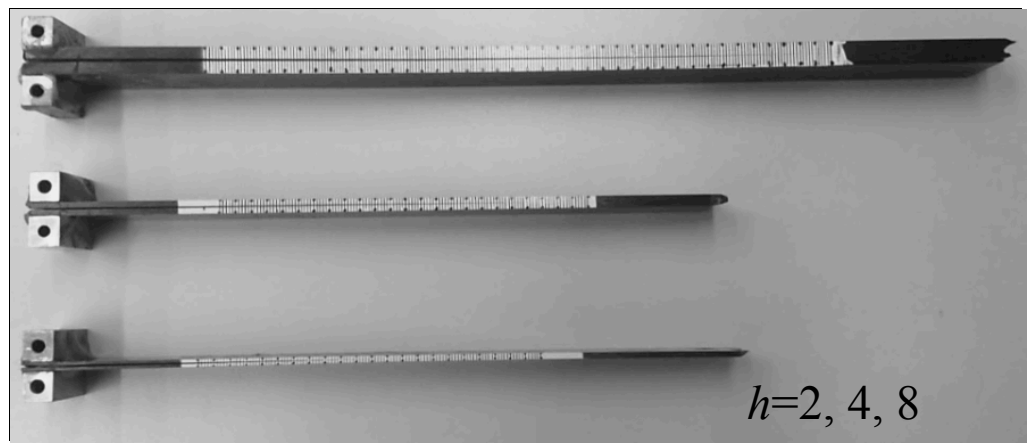

(a)

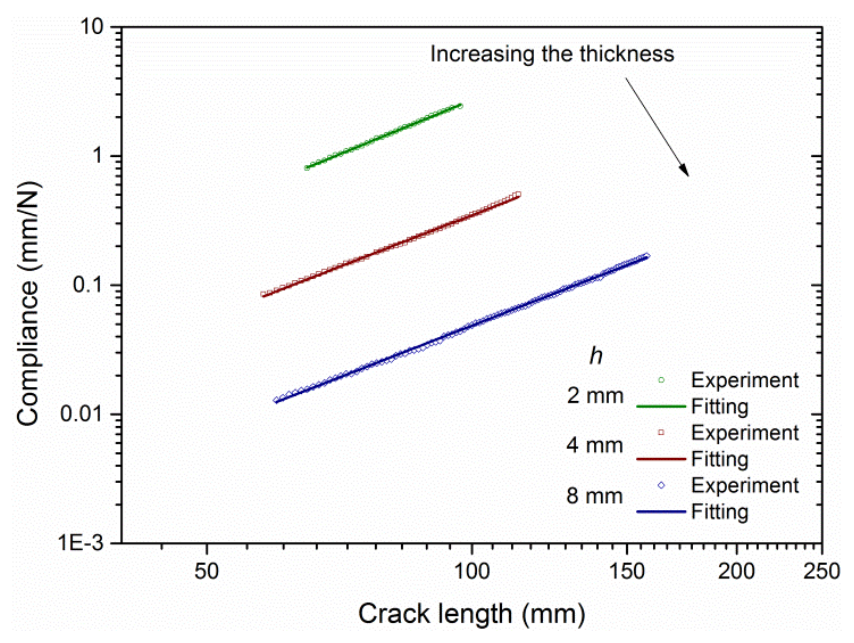

(b)

Fig. 3. (a) Photograph of representative DCB specimens tested under fatigue loading, (b) Compliance curves as a function of crack length measured in the fatigue experiments on the specimens of different thicknesses.

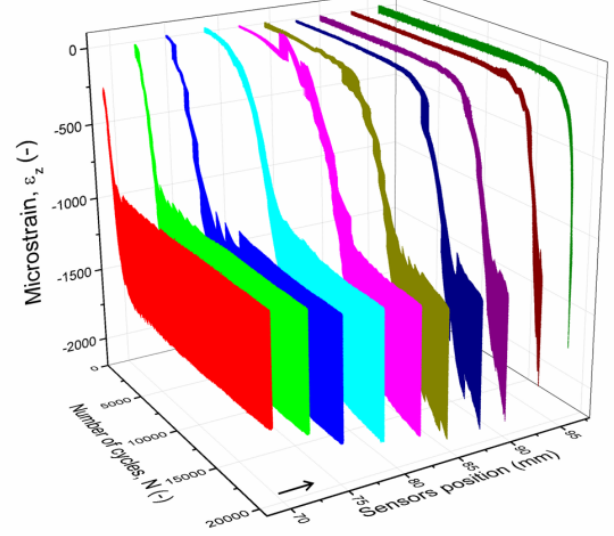

(a)

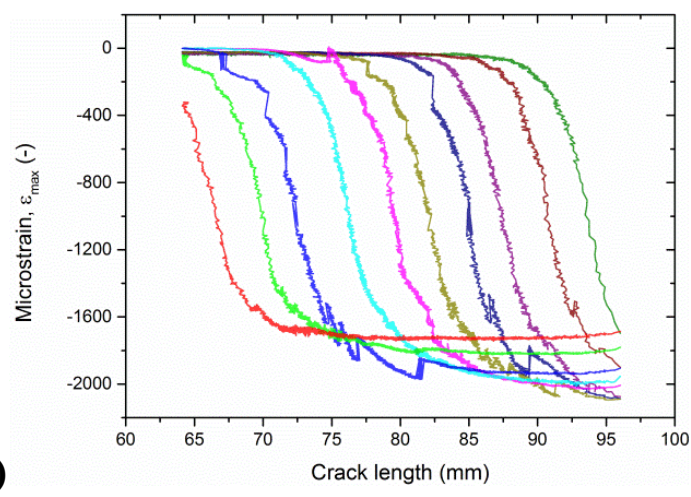

(b)

Fig. 4. (a) Recorded strain data with an array of ten FBG sensors during the entire fatigue experiment on the specimen $h=2 \mathrm{~mm}$. The arrow indicates the direction of fatigue crack growth within the sensors array, (b) Corresponding maximum cyclic strains. 


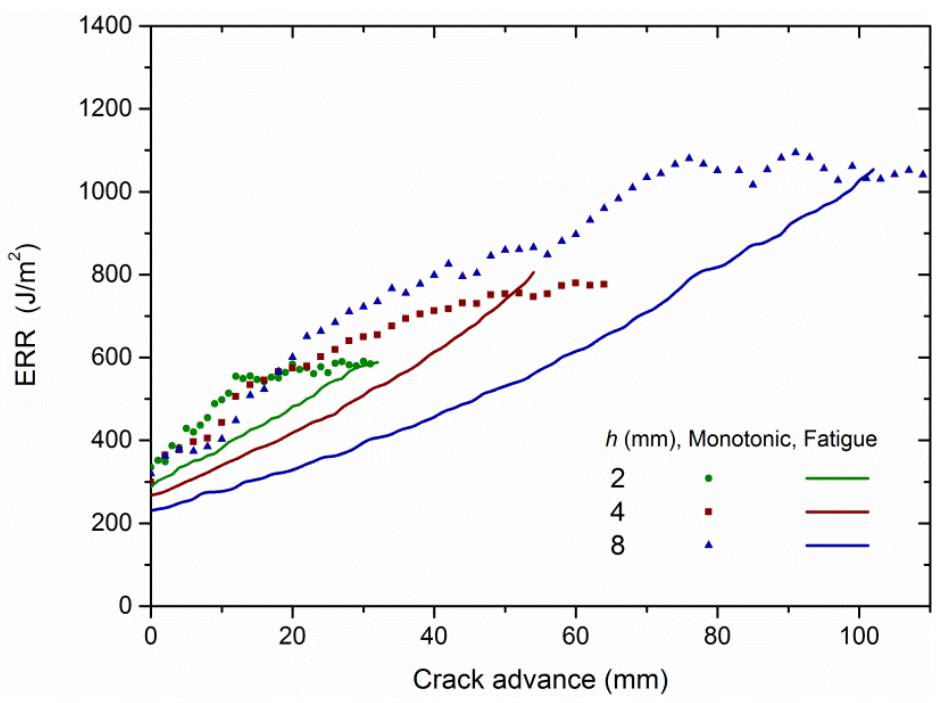

Fig. 5. Maximum cyclic ERR as a function of fatigue crack growth in the specimens of different thicknesses, together with the corresponding R-curves measured in the monotonic tests.

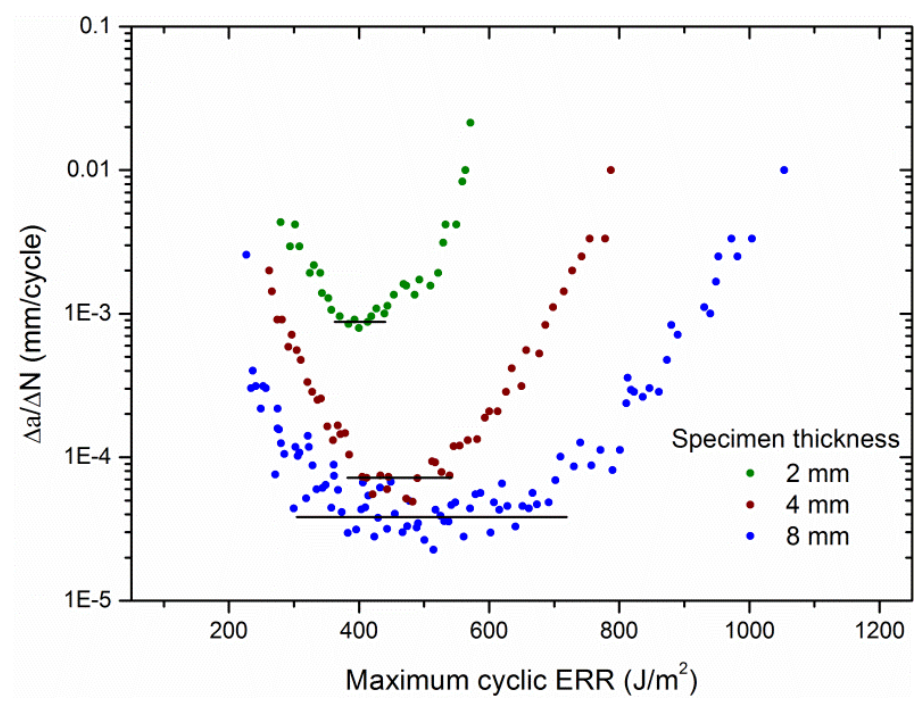

Fig. 6. Fatigue crack growth rates obtained from the specimens of different thicknesses. The horizontal lines indicate averaged constant crack speed in phase II of fatigue delamination. 


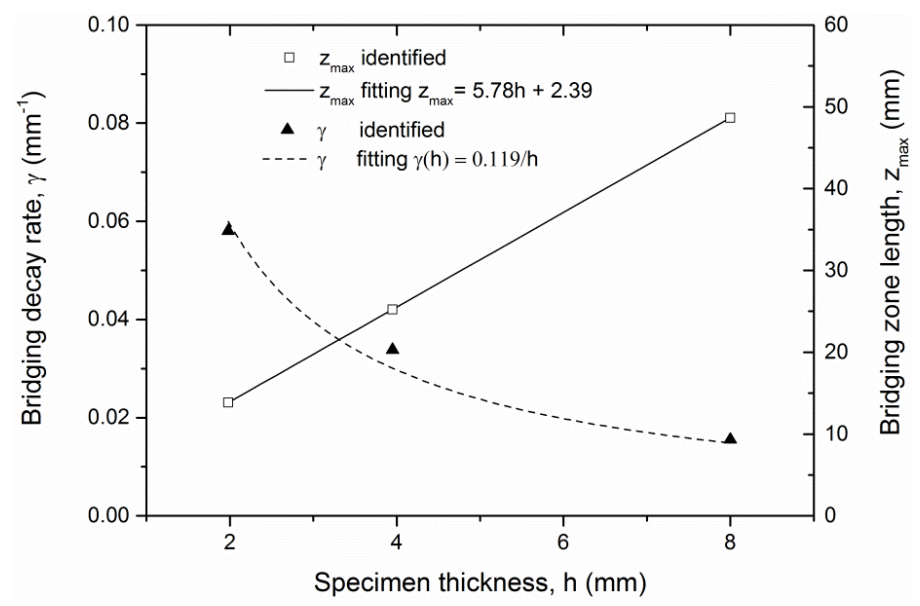

Fig. 7. The evolution of the bridging zone length, $z_{\max }$, and the nonlinear rate of tractions decay, $\gamma$, in terms of specimen thickness.

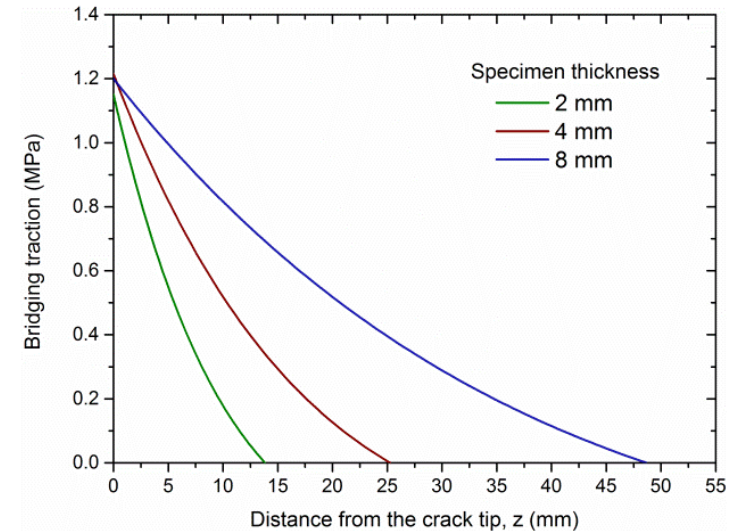

(a)

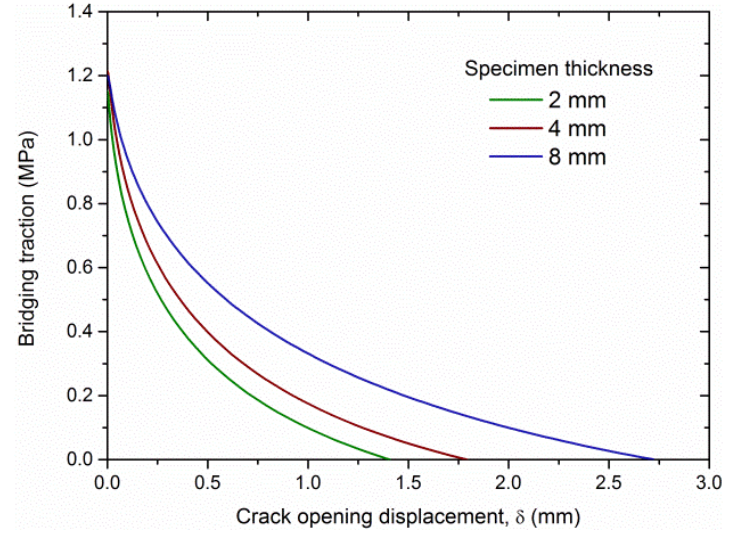

(b)

Fig. 8. (a) Identified bridging tractions along the fatigue delamination crack in the specimens of different thicknesses, (b) Identified bridging tractions as a function of crack opening displacement. 


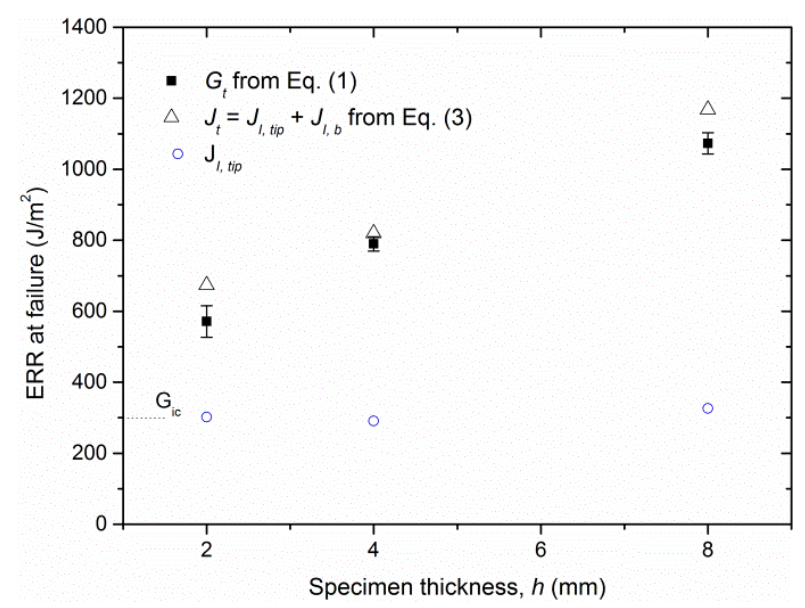

Fig. 9. Experimental data and simulations of ERR, at the end of fatigue experiments, of specimens with different thicknesses.

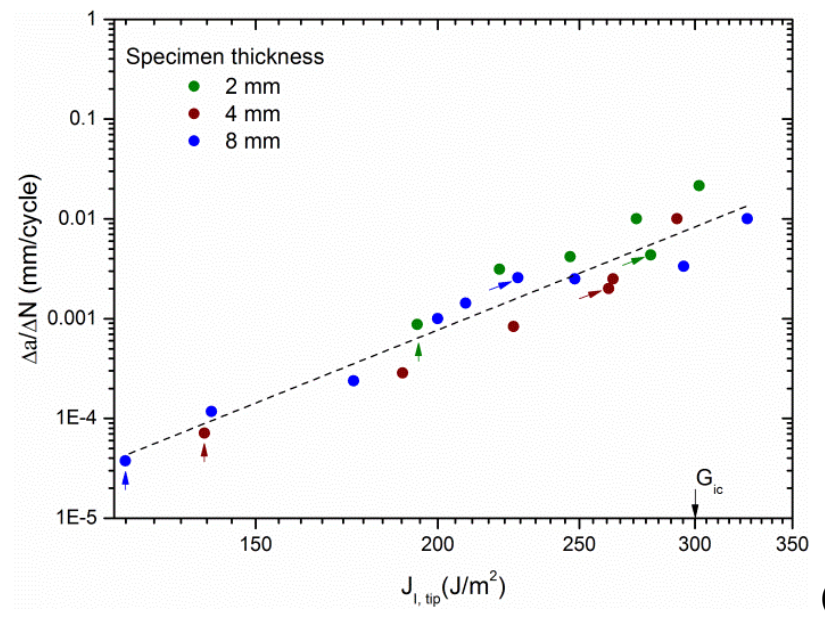

(a)

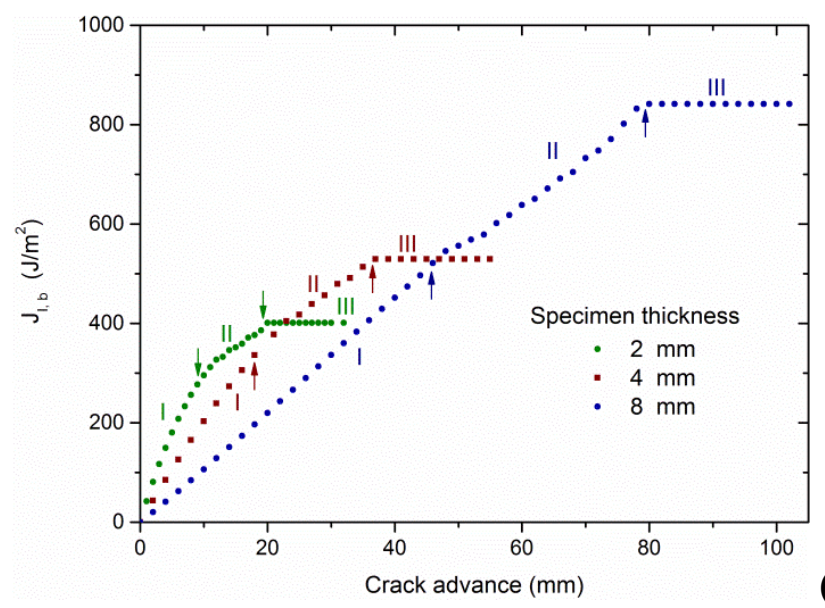

(b)

Fig. 10. (a) Crack growth rate as a function of the identified $J_{I, t i p}$ values for the specimens of different thicknesses. Inclined arrows indicate data corresponding to the first crack increment and vertical ones the average constant speed in phase II (Fig. 6), 
(b) Computed evolution of bridging ERR during the fatigue crack growth. The different propagation phases are indicated by vertical arrows. 